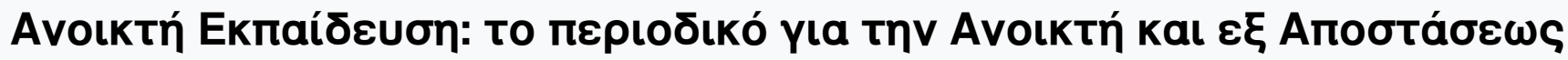

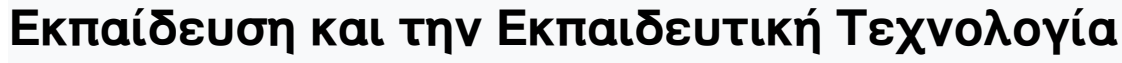

Tón. 15, Ap. 1 (2019)

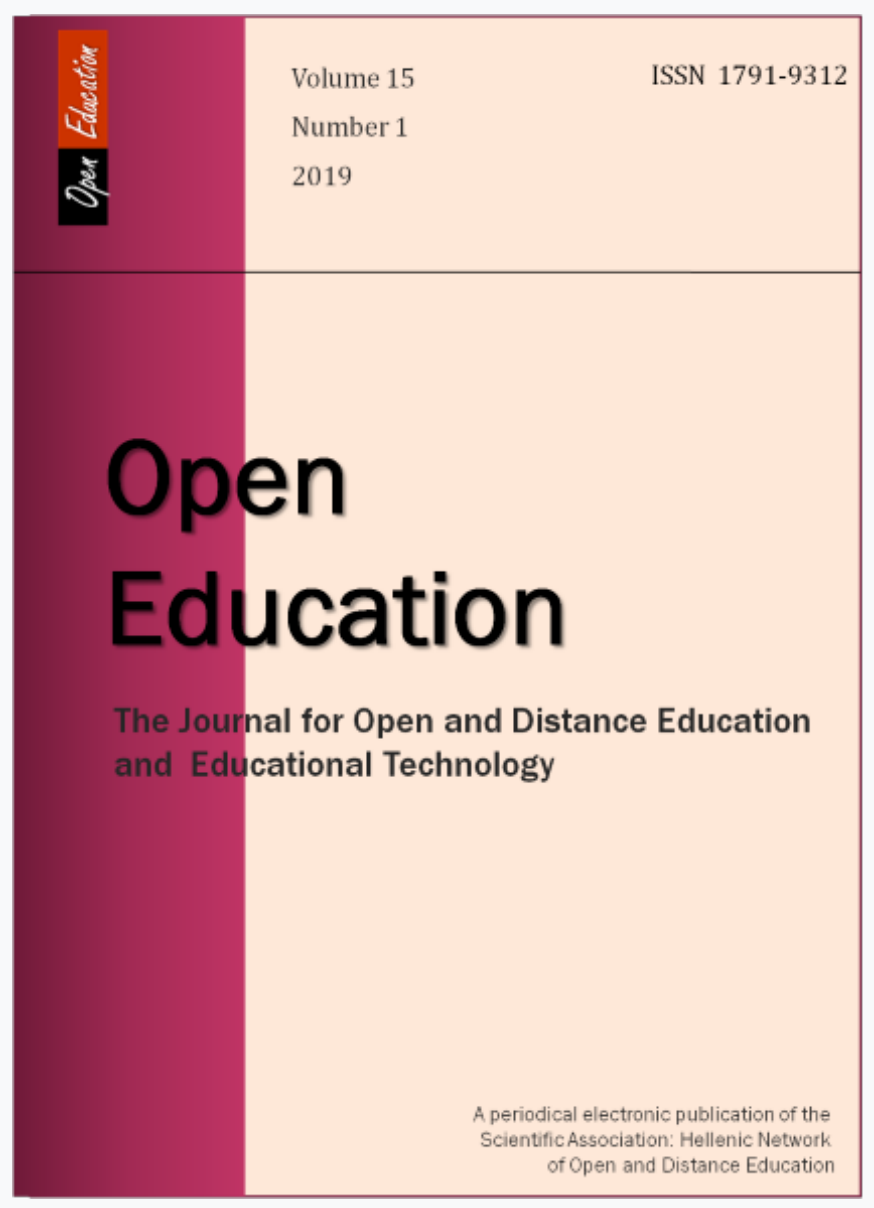

Developing a student support system at the Hellenic Open University

Antonis Lionarakis, Maria Niari, Anna Apostolidou, Kiriaki Sfakiotaki, Efi Ligoutsikou

doi: $10.12681 /$ jode.21089

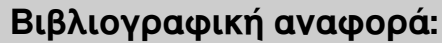




\title{
Developing a student support system at the Hellenic Open University
}

Antonis Lionarakis

PENER research team alionar@eap.gr

Maria Niari

PENER research team niarimaria@gmail.com

Anna Apostolidou

PENER research team annapostolidou@gmail.com

Kiriaki Sfakiotaki

PENER research team sfakkor@gmail.com

Efi Ligoutsikou

PENER research team efilig2@yahoo.gr

\begin{abstract}
Academic support for students in the field of open and distance education is a multifaceted and decisive parameter for enhancing and promoting student engagement and academic progress. The need to develop an effective support system for students at the Hellenic Open University is maintained by a wide array of research findings according to which there are diverse categories of learners with differentiated needs and levels of online participation. This paper describes the distance learning support system developed in the framework of the research project "PENER EAP 2016" entitled "Academic support system for students of tertiary open and distance education" which is funded by the Hellenic Open University. In this paper, we briefly present the key research findings from the literature review and the main areas where, according to the bibliography, student support is most needed. Subsequently, we describe the methodology and findings from the semi-structured interviews with the students and we describe the development of the support system for distance learning students. Concluding, we present the tele-support platform and the digital tools developed within the framework of the research project.
\end{abstract}

\section{Keywords}

student support, open and distance education, educational needs, digital platform and tools.

\section{Introduction}

The field of open and distance education has demonstrated a great interest in student support in recent years, since it has become clear that the wager of its successful integration into the 21 st century educational landscape depends to a large extent on 
the ways in which educational institutions recognize and support the particular needs of heterogeneous groups of students. Since the last decade the issue of quality student support, has occupied the interest of leading theorists in the field of distance education (Tait, 2003a, 2003b; Sewart, 1993; Keegan, 2003).

In the work of these researches, we may trace several definitions of support that reflect earlier phases of open and distance learning, particularly in Europe, as well as the transformations that the notion has undergone over time due to theoretical and technological progress. The emphasis on support was a strong priority for distance teaching universities compared to the conventional higher education (Tait, 2000), but did not always receive due attention based on its importance for the successful attendance at them. Various support guides for students were drafted in this phase (Northedge, 2001 [1990]), which were however soon discredited because of the rapid changes occurring in the field of open and distance learning and technology in recent years.The fragmentation of earlier approaches to the issue of student support has recently led to more systematic efforts of this complex issue investigation (Simpson, 2000; Thorpe, 2002), which have already begun to bear research fruit.

The research project "Academic support system for students of tertiary open and distance education", carried out by the Hellenic Open University (H.O.U.), aims at the theoretical foundation, design and creation of an integrated system of focused training support provision for undergraduate and postgraduate students in tertiary open and distance education in Greece. The concept is derived from international practice and relevant literature on an integrated system of services and methods for supporting students in an open and distance learning environment (Altbach, 2010; Whiteford, Shah, \& SidNair, 2013).

The student support system proposed by this study is potentially useful for all tertiary institutions, but especially for open \& distance universities, and presents a number of strategic advantages:

- promotes openness, i.e. the accessibility of mass higher education to students, mitigating the problems arising from the educational profile of people who choose to study at open universities (long time away from education/training, lack of training, lack of basic academic skills, lack of collaborative learning culture)

- promotes autonomous and self-directed learning, equipping students with tools and materials that enhance the independent and heuristic path towards knowledge

- promotes digital training (both the use of the platform and its content: digital tools, polymorphic educational material, digital seminars and so on)

- highlights the great importance of student support, particularly for 'peripheral' countries, which often reproduce 'metropolitan' educational models, and thus present greater needs for cultural adaptation of education and support tools

- tackles a series of problems arising from open and distance learning methodology (physical distance, lack of personal contact \& support, lack of intermediate infrastructures) and the philosophy of openness (entrance without examination, access for all etc.).

\section{Aim}

The aim of the ongoing research project "Academic support system for students of tertiary open and distance education", carried out by the Hellenic Open University is to develop an integrated student support system in the learning community of a distance learning tertiary institution. 
The secondary objectives of the research project were:

- to locate and address students' educational needs

- to gather good practices and relevant literature findings

- to develop a digital platform for training, education and support.

\section{Methodology}

In order to meet the expectations, the research design the following phases:

- Extensive literature review

- Semi-structured interviews in order to investigate the particular educational needs of the students in open and distance education

- Development of a theoretical model of training, education and support

- Development of a model online platform for training and support, i.e. a digital environment where each student can enter and navigate freely to reach the information, tools and educational material necessary for each stage of study in an open and distance learning environment.

- A pilot survey on a selected sample of students to provide feedback.

- Two expert focus groups to evaluate and provide feedback.

- Incorporation of feedback on both the theoretical framework and the platform itself.

\section{Literature review}

The PENER EAP 2016 research team collected and reviewed papers, journals, articles, proceedings, books and university guides, both printed and digital, in Greek and in English. Most recent and more sited papers were preferred. In addition, the research team preferred journals and proceedings from conferences with blind peer reviewing process.

The key concepts that were at the center of the literature review included: academic student support, organization/management of study, academic writing, cultivation of cognitive skills, collaborative learning, communities, digital tools, psychological support.

The findings from the literature review, which derived from teamwork of all research team members, are presented in the next section.

\section{Semi-structured interviews}

In the context of exploring students' needs, interviews were conducted in order to collect data for the following thematic categories which constitute the main pillars of the student support system, in distance learning environments. The interviews focused on the following thematic categories: 1. Introduction to university studies in an open and distance learning environment, 2. Organization of studying, 3. Academic writing, 4. Digital tools, 5. Communities and collaborative learning.

The interviews were conducted with the participation of four postgraduate students, who attended a graduate program on Greek and European culture at the HOU. The interviews aimed at recording the needs or obstacles they face from the beginning of their studies through completion at the Hellenic Open University. The interviews were concluded within two days and were based on an interview guide, which consisted of open-ended questions. No time limit was set during the interviews, in order to have the opportunity of receiving full, clear and comprehensive answers.

More specifically, the interviews were based on a cross sectional survey design, as this method gives evidence at a specific time, and the researchers investigate current attitudes and opinions. In addition, this method measures the needs for educational 
processes and services resulting from curricula. The focus group interviews in survey research have been chosen as a small group of people (four postgraduate students), who were the sample of these interviews. The interviews were based on open - ended questions which gave the opportunity to the sample to answer without any commitments. This method was considered appropriate for this specific project, as the interviewer does not know the possible answers and explores the options more effectively. The method of appropriate sampling was applied in selecting the sample and in particular the avalanche method. Each student took part in that interview after being informed by the first participant and encouraged among themselves to participate in this procedure. Also, none of the participants were prepared for the content of the interviews in order for their answers to be spontaneous and not preceded by conciliation. The whole procedure of the interviews was video recorded. The findings were categorized and analyzed following standard thematic analysis and the most significant data are presented in this paper.

In addition, an interview with the director of the Counseling and Psychological Support Center of the Hellenic Open University was also conducted, based in his expertise on the field of students psychological support.

\section{Findings}

\section{Literature review}

The literature review demonstrated that the issue of student support quality in the field of open and distance learning has a prominent position in the research orientation of both the international scientific literature, numerous Greek and foreign journals and several thematic conferences. In particular, approaches from various disciplines such as psychology incentives highlight the urgency of setting up an integrated theoretical model of learner support in open and distance learning (Simpson, 2008) with wording efforts related theoretical proposals.

At the same time, bibliographic research has shown a keen interest in the development of electronic learning support systems and their connection to the needs of the participants (Anglelakis, Kalles \& Tryfonas, 2011), the design of digital learning communities (Rumble, 2001; Henri \& Pudelko, 2003), as well as the need for administrative support for learners through web sites and related digital sites (Jones \& Meyer, 2012; Kishore, 2014).

Essentially, the research effort of recent years has reduced student support to a major parameter of modern open and distance learning, a finding and necessity that is also being promoted by the OpenPraxis magazine of the International Council for Open and Distance Education (2014). Indeed, corresponding international surveys point to the breadth and importance of overall student support with relevant references and research work in the Council of Higher Education Guide (2014). At the same time the research interest in supporting students' potential based on the available digital capabilities of the time is also updated by recent relevant studies (Moe-Pryce, 2012) as well as analytical guides (Butcher \& Wilson-Strydom, 2013; Butcher \& Hooser, 2014) as quality parameters provided online higher education.

The literature review has also shown that a comprehensive and applied model of student support is potentially useful for all tertiary institutions and especially for open and distance universities, as a dimension is distinguished between the learning profile of adult learners entering the above learning environments and high academic requirements to which they are called upon to respond. The above finding of the gap may lead the trainee to insufficient information and self-management of the study, to a limitation on the cultivation of specific cognitive skills, eventual interruption of 
studies, low self-esteem and frustration, low performance etc (Hurd, 2000; Stevens, 2009; Clair, 2015).

The fragmentation of existing approaches to student support provides the basis for more targeted efforts focusing on the student's own needs. Essentially, the available studies concern individual tools and technological applications without concern for learning, psychological and other peculiarities of academic support within an integrated support model. Studies on digital communities empirically map emerging student needs, their administrative support, quality assurance (Simpson, 2008; Jones \& Meyer, 2012; Kishore, 2014), but they do not guarantee a systematic and methodical support for the student.

In fact, the ongoing research project examines, among other things, the existing research data regarding the most comprehensive educational culture in Greece, focusing mainly on the educational culture of open and distance education institutions and the available support mechanisms. References to particular characteristics of student groups (gender, beliefs, etc.) in relation to the drop-out rate and special support needs have been the subject of study, as well as the importance of supporting students, particularly countries of the "periphery", which often reproduce educational models and thus present greater needs, as has been found, for example, in the research-rich example of India and other Asian countries.

However, the provision of information, explanations and tools as well as the interaction in the field of distance learning is considered by fireld experts as a fundamental element in the completion of studies (Jara, 2006). In addition, parameters such as educational culture and the specific characteristics of higher education institutions providing distance education largely influence the support and strategic choices that institutions make for the benefit of their students.

This literature review has gradually led to the development of a training and support model with subcategories according to the needs expressed by students of the Hellenic Open University during the small-scale empirical investigation under the PENER project (Spring 2017). In essence, this combination of students' training needs and open and distance learning requirements highlight the importance and timeliness of the current PENER EAP 2016 research program both theoretically and practically, since it offers a pilot system of support for students responding to a series of inherent weaknesses in distance learning.

The following seven individual thematic categories that constituted the theoretically documented model of training, education and support for distance learning students are:
A. Introduction to university studies in an open and distance education environment
B. Organization of study
C. Academic writing
D. Post graduate studies and research (exclusively for post graduate students of higher education)
E. Digital tools
F. Community \& Collaborative Learning
G. Psychological Support

\section{Interviews:}

The following key points emerged from the interviews of the undergraduate participating students, concerning the specific thematic categories in order to draw conclusions about the learning needs of students in distance learning environments. 


\section{Introduction to University Studies in an open and distance education environment.}

Undergraduate students find differences between conventional and distance learning. Keeping timetables is an element of diversity in relation to their capabilities in conventional educational environments. Difficulties encountered in managing time and organizing it in order to follow the timetable. Their expectations from attending courses in the Hellenic Open University were covered and for three of them were also exceeded. They managed to acquire knowledge, to deal with their studies that were for many of them an 'unreachable' dream, and support themselves in a professional frame. They initially faced difficulties because they were not familiar with the distance education model, an element that became apparent when they had to study in the distance education framework or to use bibliographic references. These students surpassed their difficulties with the extremely important contribution and support by tutors or coaching, and collaborating with fellow students, monitoring forums and observing debates of co-students. In addition, they seek for detailed feedback concerning their written assignments during their learning path. The students themselves emphasized that teaching in a distance education environment, is impossible to have any similarity to teaching in a conventional university. In addition to the above elements, there is a great disagreement in the way the bibliographic references are supposed to be presented in the assignments, because of different preferences of tutors. Some of them insisted on the APA system. Others insisted on using Harvard or Oxford system. They considered it important, during the face-toface meetings, to discuss issues related to improving the content of their studies. In addition, the students particularly emphasized the lack of a common line in the teaching method by tutors, as they have adopted the perception that tutors methods are not always adapted to their learning needs.

\section{Organization of Study}

Students come across with significant difficulty concerning timetables, particularly in conjunction with the extra literature for their studying, although they recognize the need for a timetable. In addition, it is noticed that they prefer to organize their archives primarily in paper form, while at the same time they make summaries and notes on the teaching material. They considered the guidance they received from their tutors as extremely assisting and targeted, but on the other hand they are notably worried about the credibility of online sources and they look for the approval of specific websites from their tutors, which are undoubtedly scientifically up-to-date. Although the internet as means for gaining information offers many possibilities, they consider that it would greatly help to use specific digital libraries. In addition, students wanted the additional resources to be integrated into the teaching material or to be embedded in studying platforms.

\section{Academic Writing}

The amount of teaching material combined with the inability to daily contact their tutors created a serious problem in the "management" of this material. Although students believe that tutors support them through provided instructions in order to adapt to academic writing, it was reported that if they do not have a basic infrastructure concerning their learning object, it would be extremely difficult to move forward and complete their learning path. They also reported the lack of a specific way of presenting bibliographic references (APA, HARVARD). In addition students faced difficulty in using the digital library of HOU, as more clear and specific instructions are deemed necessary. Regarding plagiarism, they were informed either by past (not academic) experience or by tutors who highlighted the importance of this 
issue. About bibliographic references, they believe that the correct quotation of citations and references is important, as it is in fact, "half the job".

\section{Digital Tools}

It is remarkable that undergraduate students mentioned internet, word, excel, power point, as digital tools. In addition, they prefer to directly communicate with each other rather than consult blogs, as they are particularly skeptical and feel they may be misled or become more anxious. They also suggested that the existence of a platform would be very useful for them as they would be able to submit their questions as long as the person who gives the information, and answers, not necessarily an academic, is considered an 'expert'. This is viewed as a safety net for the information reliability and credibility. In addition, they expressed the necessity of videoconferencing as it offers the opportunity to students who live far away, to attend group meetings without incurring extra costs. More specifically, students expressed the idea that "the existence of teleconferencing is a significant factor in determining how conventional or how alternative a university that offers open is and distance learning is".

\section{Community and collaborative learning}

The undergraduate students considered that the existence of collaborative work is not possible under the present structures and circumstances in H.O.U., but not only due to distance among students and tutors, but also because of the fact that no one can offer at the same time the same quantity and quality of work. However, it was considered that in the framework of H.O.U., tutors must somehow encourage co-operation among students. Undergraduate students wish to participate in their learning experience in a more experiential and interactive way as this way of learning is considered as more effective in achieving their expected learning goals. Finally, factors that make them feel like members of the academic community are the high level of education, the fact that teachers are "potential colleagues", the fact that most teachers also teach in conventional universities, the existence of fellow students, their academic identity and building facilities.

\section{Description of the platform and digital tools}

The support system is mainly digital. It consists of the tele-support platform based mainly on asynchronous support and includes modern communication services. The platform used is the Moodle platform:

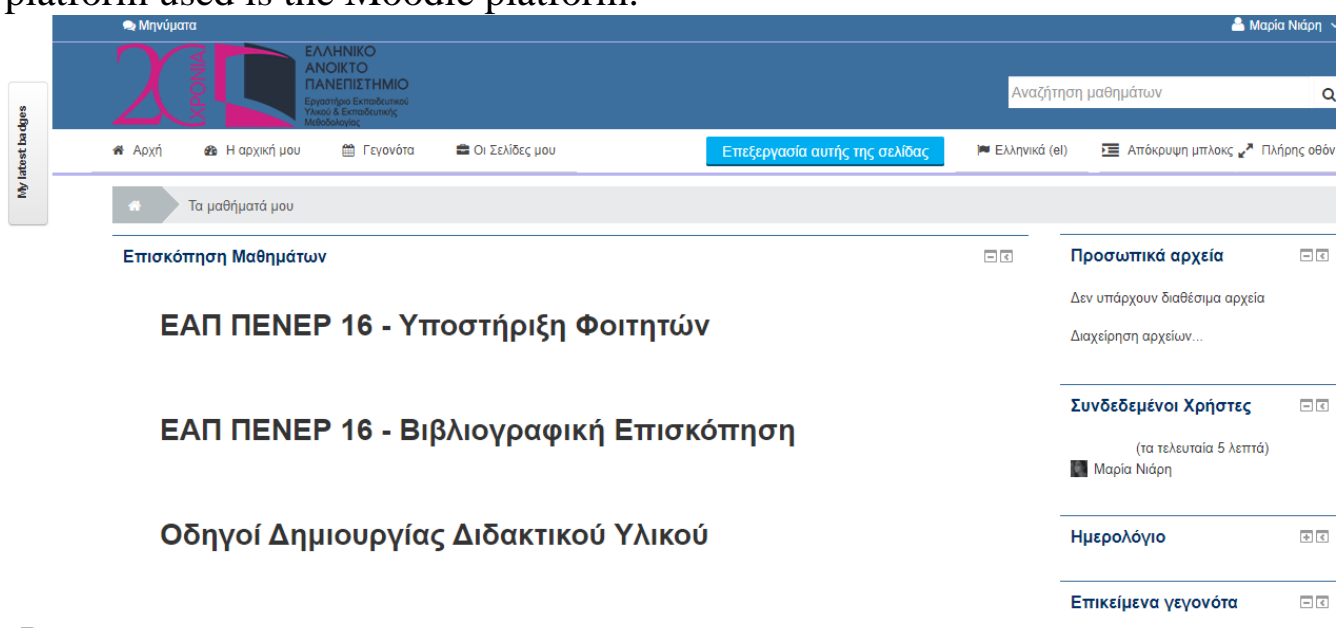

Figure 1. The tele-support EAP platform

The system is designed to include a variety of narrative styles and polymorphic support material for all the thematic sub-categories. Educational videos and animation 
have been used, as well as many presentations with a combination of audio and visual material. The research team has produced a number of different learning scenarios, aiming at targeted information provision, and offering alternative ideas for students.

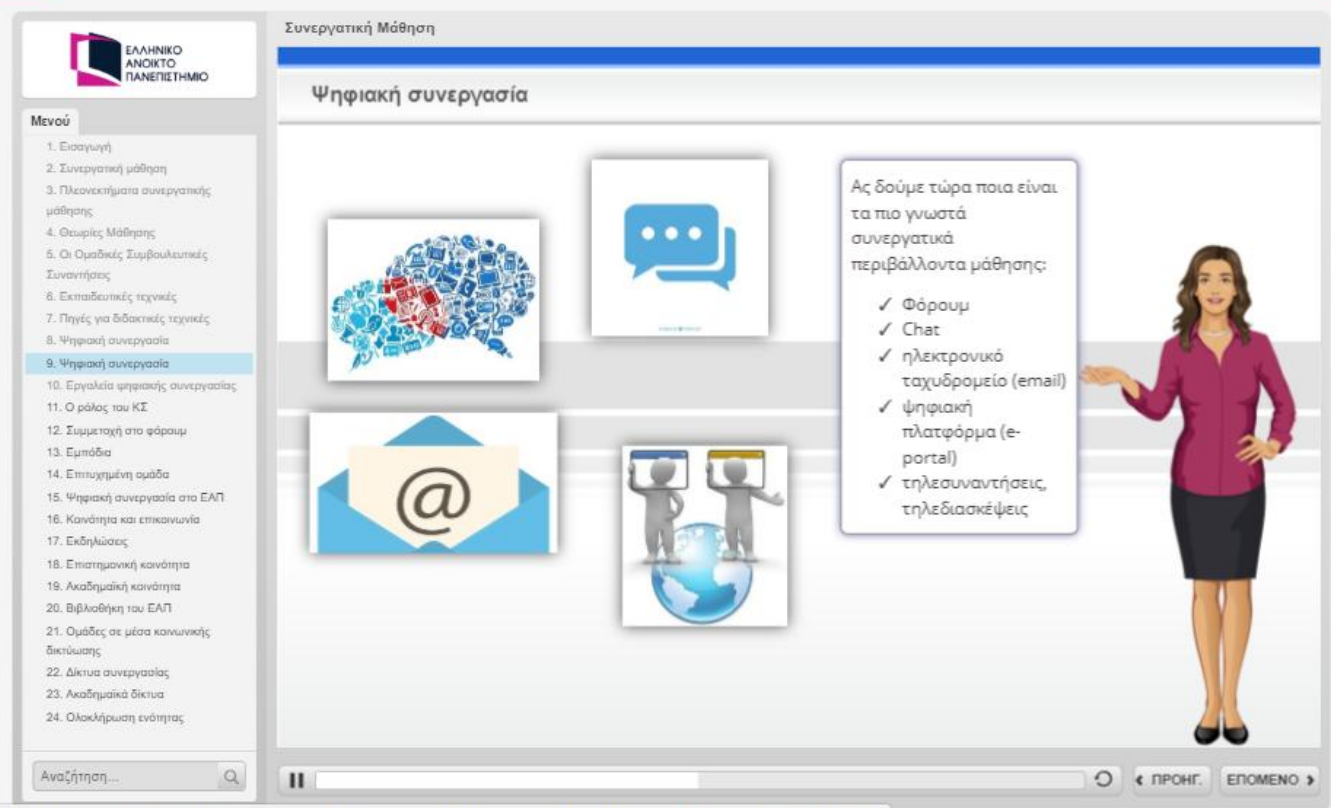

Figure 2. Example of an animated scenario

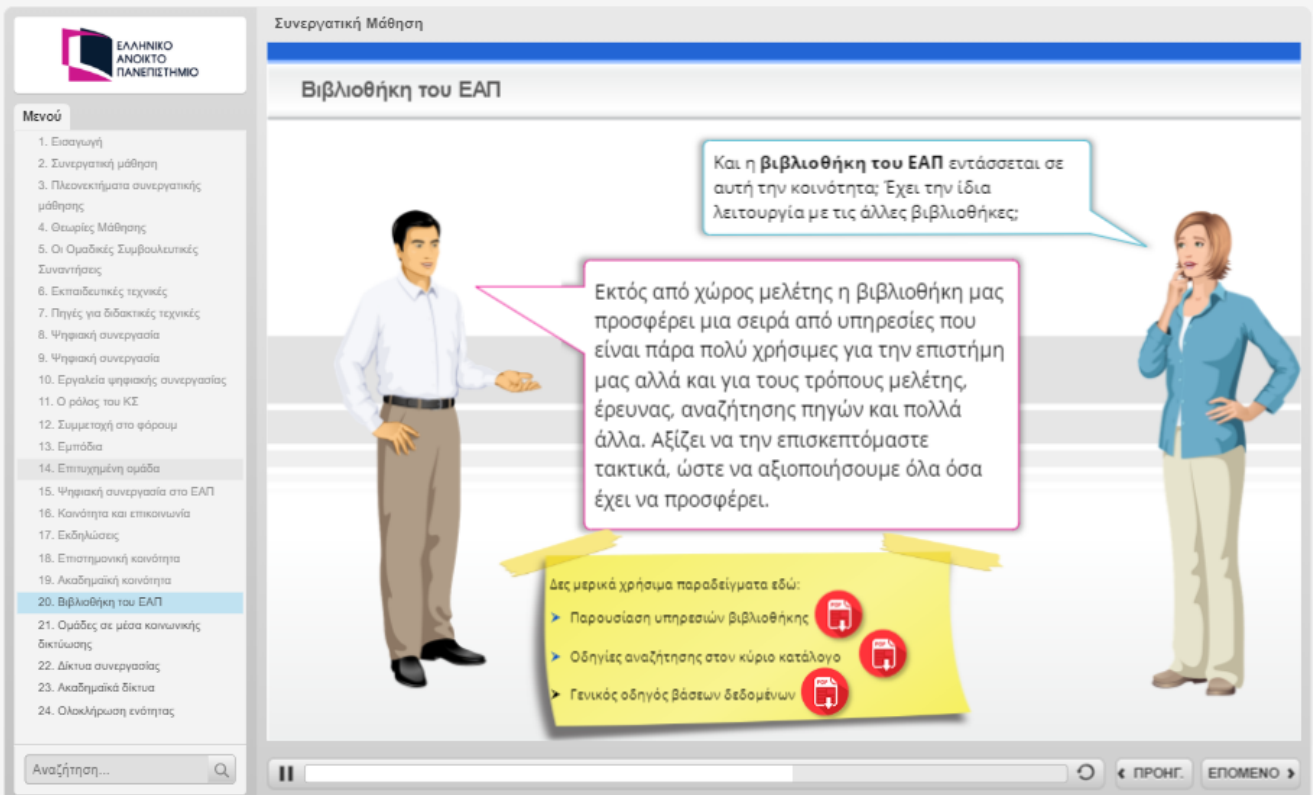

Figure 3. Example of an animated dialogue

The user enters the platform with a personal password and can navigate freely in seven different digital spaces (thematic areas), where the support tools are placed per topic category. Open access to all posted documents as well as collaborative communication tools (such as discussion forums) are provided. 


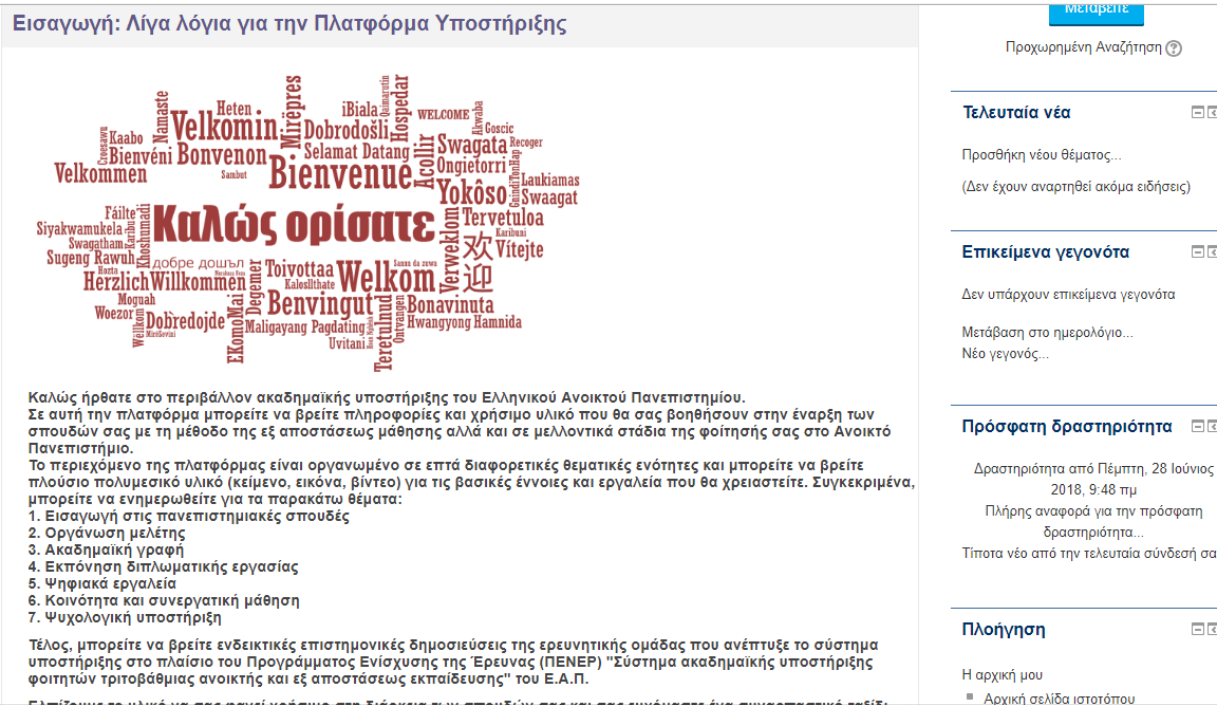

Figure 4. Introduction to the support platform

Each category is characterized by autonomy in the way of narrative, but there are links between issues related to more than one theme and thematic overlaps. The majority of the tools are in Greek, but there are also open educational resources in English (videos, podcasts, selected papers). The tools developed by the research team draw on existing valid scientific resources, compile available information and provide targeted responses to key support issues that have emerged from recent years, as well as from the empirical research conducted in the context of PENER EAP-16 in undergraduate and postgraduate students of the Hellenic Open University.

In addition, according to the pilot test (in progress), the design of the platform provides for the progressive enrichment of the tools by the tutors who will use the platform along with their students, as well as an open space for gathering proposals from all users and interested parties in order to keep a detailed record for future improvements .

\section{Conclusion}

The need to implement a system of support for studies at the Hellenic Open University resulted from the research findings, as well as from the literature review. Distance learning students in Greece are often individuals who have moved away from formal education for long periods of time, and only in a few cases have a prior acquaintance with distance learning methodology. Given the professional and personal commitments faced by students, due to the age and social-psychological phase in which they begin their studies in H.O.U., a great difficulty of coping with content and structure of their chosen educational programs is often expressed. Additional challenging factors are the lack of personal contact and the long response time of both fellow students and tutors, leading to a situation where they feel unable to complete their studies without the appropriate support.

The provision of information, explanations and tailor-made tools in the context of distance learning is considered as a fundamental element in the completion of studies. The need for interaction and communication is also highlighted from both research findings and students themselves.

For this reason, an integrated support system for students of the Hellenic Open University was designed and implemented by the research team of PENER EAP 2016. Upon completion of the project, the results and deliverables of an integrated student support model can be effectively utilized by large and massive open and 
distance education institutions in Greece and abroad, such as open universities with large numbers of students and learning specificities, which face problems and malfunctions associated with the lack of supporting infrastructure.

Finally, it should be noted that the present descriptive framework will be enriched at the completion of the next phases of the study, and especially after the findings of empirical pilot test and feedback processing.

\section{References}

Aggelaki, M. E., Kalles, D., \& Trifona, N. (2011). Developmentofan e-learning system for distance education. In: C. Panagiotakopoulos (Ed.), Proceedings of $2^{\text {nd }}$ Conference ETPE. Patras, 2830/04/2011. Retrieved 08/072018 fromhttp://www.etpe.gr/custom/pdf/etpe1688.pdf. [in Greek]

Altbach, P. G. (2010). Access Means Inequality. International Higher Education, 61(3-5). Retrieved $26 / 03 / 2016$ http://www.mbaempresarial.com.br/mit_sloan_club/artigo_ricardobetti_bostoncollege.pdf.

Butcher, N., \& Hooser, S. (2014). A Guide to Quality in Post-Traditional Online Higher Education. Academic Partnerships.

Butcher, N., \& Wilson-Strydom, M. (2013). A Guide to Quality in Online Learning. Academic Partnerships.

Clair, D.(2015). A Simple Suggestion for Reducing First Time Online Student Anxiety, Merlot Journal of Online Learning and Teaching, 11(1). Retrieved 25/11/2015 from http://jolt.merlot.org/vol11no1/StClair_0315.pdf

Henri, F.,\& Pudelko, B. (2003). Understanding and analyzing activity and learning in virtual communities. Journal of Computer Assisted Learning, 19, pp. 474-487. Retrieved 12/10/2016 from https://hal.inria.fr/file/index/docid/190267/filename/Henri-France-2003.pdf.

Hurd, S. (2000). Distance language learners and learner support: beliefs, difficulties and use of strategies. Links and Letters 7: Autonomy in language learning 7, pp. 61-80. Retrieved 09/07/ 2018 from http://oro.open.ac.uk/21782/1/DLLLearnSupp-LinksLetters-Hurd-2000.pdf

Jones, S. \& Meyer, R. (2012). The Virtual Face of Distance Learning at Public Colleges and Universities: What Do Websites Reveal about Administrative Student Support Services? Online Journal of Distance Learning Administration. (15), 4. Retrieved 10/11/2016from http://www.westga.edu/ distance/ojdla/winter154/jones_meyer154.html.

Keegan, D. (2003). Introduction. In: Rekkedal, T, Keegan, D, Fritsch h. et al. (Eds.), The role of student support services in e-learning systems, pp. 1-6. Zentrales Institut für Fernstudienforschung: Fernuniversität Hagen. Retrieved 24/03/2015 from https://ubdeposit.fernunihagen.de/receive/mir_mods_00000362.

Kishore, S. (2014). Academic counselling in ODL: Information System for Capacity Building of Academic Counselors' in IGNOU. Turkish Online Journal of Distance Education, 15(2), 98107.

Tamilnadu,

INDIA. Retrieved07/04/2018fromhttp://files.eric.ed.gov/fulltext/EJ1043085.pdf.

Moe-Pryce, N. (2012). Is Open and Distance Learning the Key to Quality Higher Education for All? Retrieved 02/03/2015 from https://edutechdebate.org/open-and-distance-learning/are-open-anddistance-learningthe-key-to-quality-higher-education-for-all/.

Northedge, A. (2001). [(1990). The Good Study Guide. The Open University.]

Rumble, G. (2001). Just how relevant is e-education to Global educational needs? Open Learning: The Journal of Open and Distance Learning, 16(3), pp. 223-232. Retrieved 08/07/2018 from http://citeseerx.ist.psu.edu/viewdoc/download?doi=10.1.1.500.2612\&rep=rep1\&type=pdf.

Sewart, D. (1993). Student support systems in distance education. Open Learning, 8(3), pp. 3-12.

Simpson, O. (2008) Motivating learners in open and distance learning: do we need a new theory of learner support? Open Learning The Journal of Open and Distance Learning, 23(3), pp. 159170. Retrieved 24/03/2015 from http://oro.open.ac.uk/19221/2/CE977BD9.pdf

Tait, A. (2000). Planning student support for open and distance learning. Open learning, 15(3), pp. 287-299.

Tait, A. (2003a). Guest Editorial-Reflections on Student Support in Open and Distance Learning. The International Review of Research in Open and Distributed Learning, 4(1).

Tait, A. (2003b). Reflections on Student Support in Open and Distance Learning. In International Review of Research in Open and Distance Learning. Retrieved 23/03/2015 from www.irrodl.org/index.php/irrodl/article/view/134/214 
Thorpe, M. (2002). Rethinking Learner Support: the Challenge of Collaborative On-line Learning. Open Learning, 17(2), pp. 105-119.

Whiteford, G., Shah, M., \& Sid Nair, C. (2013). Equity and excellence are not mutually exclusive: A discussion of academic standards in an era of widening participation. Quality Assurance in Education, 21(3), pp. 299-310. Retreived 26/03/2015 from http://www.emeraldinsight.com/doi/abs/10.1108/QAE-Apr-2012-0020

\section{Acknowledgments}

Research project entitled "Academic support system for students of tertiary open and distance education" (PENER EAP 2016). Academic Director: Prof. Antonis Lionarakis. Research team: Aggeli Athanasia, Apostolidou Anna, Hartofylaka Antonia-Maria, Ioakimidou Vasiliki, Ligoutsikou Efi, Manousou Evangelia, Niari Maria, Papadimitriou Sofia, Sfakiotaki Kyriaki, Siakas Spyridon, Stamati Marianna, Stavropoulos Ilias, Tzilou Georgia, VerikiosVassilios. The present paper is a product of team work in collecting and reviewing the literature. 